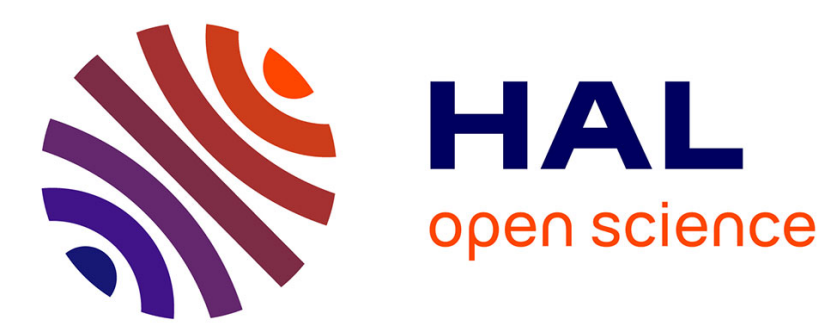

\title{
A DDVV INEQUALITY FOR SUBMANIFOLDS OF WARPED PRODUCTS
}

\author{
Julien Roth
}

\section{To cite this version:}

Julien Roth. A DDVV INEQUALITY FOR SUBMANIFOLDS OF WARPED PRODUCTS. Bulletin of the Australian Mathematical Society, 2017, 95 (3), pp.495-499. hal-01338102

\section{HAL Id: hal-01338102 \\ https://hal.science/hal-01338102}

Submitted on 27 Jun 2016

HAL is a multi-disciplinary open access archive for the deposit and dissemination of scientific research documents, whether they are published or not. The documents may come from teaching and research institutions in France or abroad, or from public or private research centers.
L'archive ouverte pluridisciplinaire HAL, est destinée au dépôt et à la diffusion de documents scientifiques de niveau recherche, publiés ou non, émanant des établissements d'enseignement et de recherche français ou étrangers, des laboratoires publics ou privés. 


\title{
A DDVV INEQUALITY FOR SUBMANIFOLDS OF WARPED PRODUCTS
}

\author{
JULIEN ROTH
}

\begin{abstract}
We prove a DDVV inequality for submanifolds of warped products of the form $I \times{ }_{a} \mathbb{M}^{n}(c)$ where $I$ is an interval and $\mathbb{M}^{n}(c)$ a real space form of curvature $c$. As an application, we give a rigidity result for submanifolds of $\mathbb{R} \times_{e^{\lambda t}} \mathbb{H}^{n}(c)$.
\end{abstract}

RÉSUMÉ. Une inégalité de type DDVV pour les sous-variétés des produits tordus. Nous donnons une inégalité de type DDVV pour les sous-variétés des produits tordus de la forme $I \times{ }_{a} \mathbb{M}^{n}(c)$ où $I$ est un interval et $\mathbb{M}^{n}(c)$ un espace modèle réel de courbure constante $c$. Nous en déduisons un résultat de rigidité pour les sous-variétés de $\mathbb{R} \times e_{e^{\lambda t}}$ $\mathbb{H}^{n}(c)$.

\section{Version francaise abrégée.}

Soit $\left(M^{m}, g\right)$ une variété riemannienne immergée isométriquement dans une variété riemannienne ambiante $\left(N^{m+p}, \bar{g}\right)$, de dimension $m+p$. Lorsque $N$ est un espace modèle simplement connexe à courbure sectionnelle constante $c$, l'inégalité suivante est vérifiée :

$$
\|H\|^{2} \geqslant \rho+\rho^{\perp}-c
$$

où $\rho=\frac{2}{n(n-1)} \sum_{i<j}\left\langle R\left(e_{i}, e_{j}\right) e_{j}, e_{i}\right\rangle$ est la courbure scalaire normalisée de $(M, g)$ et $\rho^{\perp}=\frac{2}{n(n-1)} \sum_{i<j} \sum_{\alpha<\beta}\left\langle R^{\perp}\left(e_{i}, e_{j}\right) \xi_{\alpha}, \xi_{\beta}\right\rangle$ la courbure scalaire normale (également normalisée), $\left\{e_{1}, \cdots, e_{n}\right\}$ et $\left\{\xi_{1}, \cdots \xi_{p}\right\}$ étant respectivement des bases orthonormées locales de $T M$ et $T^{\perp} M$. Cette inégalité est connue sous le nom de conjecture de DDVV car conjecturée par De Smets-Dillen-Verstraelen-Vrancken [2]. La conjecture a été démontrée récemment par $\mathrm{Lu}[$ [6] et par Ge-Tang [4] indépendamment. Plus récemment, Chen et Cui [1] ont obtenu une inégalité comparable dans le cas des espaces produits $\mathbb{S}^{n} \times \mathbb{R}$ et $\mathbb{H}^{n} \times \mathbb{R}$. Le but de cette note est d'étendre le résultat de Chen-Cui pour les sous-variétés des produits tordus $I \times{ }_{a} \mathbb{M}^{n}(c)$, c'est-à-dire $I \times \mathbb{M}^{n}(c)$ muni de la métrique $\widetilde{g}=d t^{2}+a(t)^{2} g_{\mathbb{M}^{n}(c)}$, $I$ étant un intervalle de $\mathbb{R}$ et $a: I \longrightarrow \mathbb{R}$ une fonction lisse ne s'annulant pas. Nous démontrons le résultat suivant.

Théorème 1. Soient $n>m \geqslant 2$ deux entiers. Soit $M^{m}$ une sous-variété du produit tordu $I \times{ }_{a} \mathbb{M}^{n}(c)$ avec courbure scalaire et courbure scalaire normale normalisées $\rho$ et $\rho^{\perp}$ et courbure moyenne H. L'inégalité suivante est vérifiée :

$$
\|H\|^{2} \geqslant \rho+\rho^{\perp}+\left(\frac{\left(a^{\prime}\right)^{2}}{a^{2}}-\frac{c}{a^{2}}\right)\left(1-\frac{2}{n}\|T\|^{2}\right)-\frac{2 a^{\prime \prime}}{n a}\|T\|^{2} .
$$

Nous en déduisons un résultat pour les surfaces des produits tordus $\mathbb{R} \times_{e^{4 \lambda t}} \mathbb{H}^{n}(c)$.

Corollaire 1. Soit $M^{m}$ une sous-variété complète sans bord du produit tordu $\mathbb{R} \times e_{e^{\lambda t}} \mathbb{H}^{n}(c)$ avec courbure scalaire et courbure scalaire normale normalisées $\rho$ et $\rho^{\perp}$ et courbure 
moyenne H. Si $\|H\|^{2} \leqslant \rho+\lambda^{2}$, alors

$$
\|H\|^{2}=\rho+\lambda^{2}, \quad \rho^{\perp}=0, \quad n=2 \quad \text { et } \quad\|T\|=1 .
$$

En particulier, $M$ est une surface du type $\mathbb{R} \times_{e^{\lambda t}} \gamma$, où $\gamma$ est une courbe dans $\mathbb{H}^{n}(c)$.

\section{INTRODUCTION}

Let $\left(M^{n}, g\right)$ be a $n$-dimensional Riemannian manifold isometrically immersed into a $(n+p)$ - dimensional Riemannian manifold $\left(N^{n+p}, \bar{g}\right)$. When the ambient space is a real space form of constant sectional curvature $c$, we have the following pointwise inequality

$$
\|H\|^{2} \geqslant \rho+\rho^{\perp}-c,
$$

where $\rho=\frac{2}{n(n-1)} \sum_{i<j}\left\langle R\left(e_{i}, e_{j}\right) e_{j}, e_{i}\right\rangle$ is the normalized scalar curvature of $(M, g)$ and $\rho^{\perp}=\frac{2}{n(n-1)} \sum_{i<j} \sum_{\alpha<\beta}\left\langle R^{\perp}\left(e_{i}, e_{j}\right) \xi_{\alpha}, \xi_{\beta}\right\rangle$ is the normalized normal curvature of the immersion, where $\left\{e_{1}, \cdots, e_{n}\right\}$ and $\left\{\xi_{1}, \cdots \xi_{p}\right\}$ are respectively orthonormal frames of $T M$ and $T^{\perp} M$. This inequality, known as DDVV conjecture, was conjectured by De Smets-Dillen-Verstrealen-Vrancken in [2] and proved recently by Lu [6] and by Ge-Tang [4] independently. More recently, Chen and Cui generalized this inequality in the setting of product spaces $\mathbb{S}^{n} \times \mathbb{R}$ and $\mathbb{H}^{n} \times \mathbb{R}$.

In this note, we extend Chen-Cui result by proving a DDVV inequality for submanifolds o warped products $I \times{ }_{a} \mathbb{M}^{n}(c)$ where $I \subset \mathbb{R}$ is an interval and $a: I \longrightarrow \mathbb{R}$ is a nowhere vanishing smooth function. We denote by $\partial_{t}=\frac{\partial}{\partial t}$ the unit vector field tangent to the factor $I$. We prove the following result.

Theorem 1. Let $M^{m}$ be a submanifold of the warped product $I \times{ }_{a} \mathbb{M}^{n}(c)$ with normalized scalar and normal scalar curvatures $\rho$ and $\rho^{\perp}$ and mean curvature $H$. Then, we have

$$
\|H\|^{2} \geqslant \rho+\rho^{\perp}+\left(\frac{\left(a^{\prime}\right)^{2}}{a^{2}}-\frac{c}{a^{2}}\right)\left(1-\frac{2}{n}\|T\|^{2}\right)-\frac{2 a^{\prime \prime}}{n a}\|T\|^{2},
$$

where $T$ is the part of $\partial t$ tangent to $M$.

Remark 1. Note that, of course, we recover the DDVV inequality of [1] for product spaces $\mathbb{S}^{n} \times \mathbb{R}$ and $\mathbb{H}^{n} \times \mathbb{R}$ as well as for $\mathbb{R}^{n+1}$ by taking $a=1$, but we also recover the inequality for space forms. Indeed, $\mathbb{S}^{n}$ and $\mathbb{H}^{n}$ can be expressed in term of warped products. Namely, we have

(1) $\mathbb{S}^{n}=[0,2 \pi] \times{ }_{a} \mathbb{S}^{n-1}$ with $a(t)=\sin (t)$. Hence the inequality of Theorem 1 becomes $\|H\|^{2} \geqslant \rho+\rho^{\perp}-1$.

(2) $\mathbb{H}^{n}=\left[0,+\infty\left[\times_{a} \mathbb{S}^{n-1}\right.\right.$ with $a(t)=\sinh (t)$ or $\mathbb{H}^{n}=\mathbb{R} \times_{a} \mathbb{R}^{n-1}$ with $a(t)=e^{-t}$. For both cases, the inequality of Theorem 1 becomes $\|H\|^{2} \geqslant \rho+\rho^{\perp}+1$.

\section{PRELIMINARIES}

Let $\mathbb{M}^{n}(c)$ be the simply connected real space form of dimension $n$ and constant curvature $c$. Let $I \subset \mathbb{R}$ an interval and $a: I \longrightarrow \mathbb{R}$ be a nowhere vanishing smooth function. We consider the warped $\widetilde{P}^{n+1}=I \times{ }_{a} \mathbb{M}^{n}(c)$, that the product $I \times \mathbb{M}^{n}(c)$ endowed the metric $\tilde{g}=d t^{2}+a(t)^{2} g_{\mathbb{M} n(c)}$. We denote by $\partial_{t}=\frac{\partial}{\partial t}$ the unit vector field tangent to the factor $I$. We recall (see [5] for instance) that the curvature tensor of $\left(\widetilde{P}^{n+1}, \widetilde{g}\right)$ is given by

$$
\begin{aligned}
\widetilde{R}(X, Y) Z= & \left(\frac{\left(a^{\prime}\right)^{2}}{a^{2}}-\frac{c}{a^{2}}\right)(\langle X, Z\rangle Y-\langle Y, Z\rangle X) \\
& +\left(\frac{a^{\prime \prime}}{a}-\frac{\left(a^{\prime}\right)^{2}}{a^{2}}+\frac{c}{a^{2}}\right)\left(\langle X, Z\rangle\left\langle Y, \partial_{t}\right\rangle \partial_{t}-\langle Y, Z\rangle\left\langle X, \partial_{t}\right\rangle \partial_{t}\right. \\
& \left.-\left\langle Y, \partial_{t}\right\rangle\left\langle Z, \partial_{t}\right\rangle X+\left\langle X, \partial_{t}\right\rangle\left\langle Z, \partial_{t}\right\rangle Y\right)
\end{aligned}
$$


Let $\left(M^{m}, g\right)$ be a Riemannian manifold isometrically immersed into $\widetilde{P}$. We denote by $B$ its second fundamental form and $A$ the shape operator defined for any $X, Y \in \Gamma(T M)$ and $\xi \in \Gamma\left(T^{\perp} M\right)$ by $\langle B(X, Y), \xi\rangle=\left\langle A_{\xi} X, Y\right\rangle$. Moreover, $\partial_{t}$ can be written

$$
\partial_{t}=T+\sum_{\alpha=1}^{p} f_{\alpha} \xi_{\alpha}
$$

where $T$ is a vector field tangent to $M,\left\{\xi_{1} \cdots, \xi_{p}\right\}$ is a local orthonormal frame of $T^{\perp} M$ and $f_{1}, \cdots, f_{p}$ are smooth functions over $M$. We will simply denote $A_{\xi_{\alpha}}$ by $A_{\alpha}$.

From the expression of the curvature tensor of $\widetilde{P}$, we get immediately the Gauss, Codazzi and Ricci equations for a submanifold of $\widetilde{P}$. Namley, if we denote by $R$ and $R^{\perp}$ the curvature tensor of $(M, g)$ and the normal curvature respectively, we have the following

Proposition 2.1. The Gauss, Codazzi and Ricci equations of the immersion of $M$ into $\widetilde{P}$ are respectively

$$
\begin{aligned}
\langle R(X, Y) Z, W\rangle= & \langle B(Y, Z), B(X, W)\rangle-\langle B(Y, W), B(X, Z)\rangle \\
& +\left(\frac{\left(a^{\prime}\right)^{2}}{a^{2}}-\frac{c}{a^{2}}\right)(\langle X, Z\rangle\langle Y, W\rangle-\langle Y, Z\rangle\langle X, W\rangle) \\
& +\left(\frac{a^{\prime \prime}}{a}-\frac{\left(a^{\prime}\right)^{2}}{a^{2}}+\frac{c}{a^{2}}\right)(\langle X, Z\rangle\langle Y, T\rangle\langle W, T\rangle-\langle Y, Z\rangle\langle X, T\rangle\langle W, T\rangle \\
& -\langle Y, T\rangle\langle Z, T\rangle\langle X, W\rangle+\langle X, T\rangle\langle Z, T\rangle\langle Y, W\rangle), \\
\left\langle\left(\widetilde{\nabla}_{X} B\right)(Y, Z), \xi_{\alpha}\right\rangle & =\left(\frac{a^{\prime \prime}}{a}-\frac{\left(a^{\prime}\right)^{2}}{a^{2}}+\frac{c}{a^{2}}\right) f_{\alpha}(\langle Y, T\rangle\langle X, Z\rangle-\langle X, T\rangle\langle Y, Z\rangle), \\
& \left\langle R^{\perp}(X, Y) \nu, \xi\right\rangle=\quad\left\langle\left[A_{\nu}, A_{\xi}\right] X, Y\right\rangle .
\end{aligned}
$$

The proof is straightforward form the expression of $\widetilde{R}$.

Finally, we recall that the DDVV conjecture was reduced to the following algebraic result (see [3]) proved by Lu.

Theorem ([6]). Let $n, p \geqslant 2$ be two integers and $M_{1}, M_{2}, \cdots, M_{p}$ be some $n \times n$ real symmetric and trace-free matrices. Then, we have

$$
\sum_{\alpha, \beta=1}^{p}\left\|\left[M_{\alpha}, M_{\beta}\right]\right\|^{2} \leqslant\left(\sum_{\alpha=1}^{p}\left\|M_{\alpha}\right\|^{2}\right)^{2}
$$

Now, we are able to prove Theorem 1 . 


\section{Proof of theorem 1}

First, from the definition of $\rho$ and using the Gauss equation, we have

$$
\begin{aligned}
\rho= & \frac{2}{n(n-1)} \sum_{i<j}\left\langle R\left(e_{i}, e_{j}\right) e_{j}, e_{i}\right\rangle \\
= & \frac{1}{n(n-1)} \sum_{i \neq j}\left\langle R\left(e_{i}, e_{j}\right) e_{j}, e_{i}\right\rangle \\
= & \frac{1}{n(n-1)} \sum_{i \neq j}\left(\left\langle B\left(e_{j}, e_{j}\right), B\left(e_{i}, e_{i}\right)\right\rangle-\left\|B\left(e_{i}, e_{j}\right)\right\|^{2}+\left(\frac{\left(a^{\prime}\right)^{2}}{a^{2}}-\frac{c}{a^{2}}\right)\right. \\
& \left.+\left(\frac{a^{\prime \prime}}{a}-\frac{\left(a^{\prime}\right)^{2}}{a^{2}}+\frac{c}{a^{2}}\right)\left(\left\langle T, e_{i}\right\rangle^{2}+\left\langle T, e_{j}\right\rangle^{2}\right)\right) \\
= & \left(\frac{\left(a^{\prime}\right)^{2}}{a^{2}}-\frac{c}{a^{2}}\right)+\frac{1}{n(n-1)}\left(n^{2}\|H\|^{2}-\|B\|^{2}+2(n-1)\left(\frac{a^{\prime \prime}}{a}-\frac{\left(a^{\prime}\right)^{2}}{a^{2}}+\frac{c}{a^{2}}\right)\|T\|^{2}\right)
\end{aligned}
$$

Now, we set $\tau=B-H g$ the traceless part of the second fundamental form. Clearly, we have $\|\tau\|^{2}=\|B\|^{2}-n\|H\|^{2}$. Hence we get

(4) $\rho=\left(\frac{\left(a^{\prime}\right)^{2}}{a^{2}}-\frac{c}{a^{2}}\right)\left(1-\frac{2}{n}\|T\|^{2}\right)-\frac{2 a^{\prime \prime}}{n a}\|T\|^{2}+\|H\|^{2}-\frac{1}{n(n-1)}\|\tau\|^{2}$.

Moreover, for any $\alpha \in\{1, \cdots, p\}$, we set $S_{\alpha}: T M \rightarrow T M$ the operator defined by $\left\langle S_{\alpha} X, Y\right\rangle=\left\langle\tau(X, Y), \xi_{\alpha}\right\rangle$. Obviously, we have $S_{\alpha}=A_{\alpha}-\left\langle H, \xi_{\alpha}\right\rangle$ Id and $\left[A_{\alpha}, A_{\beta}\right]=$ $\left[S_{\alpha}, S_{\beta}\right]$. From the Ricci Equation, given in Proposition 2.1, we have

$$
\rho^{\perp}=\frac{1}{n(n-1)} \sqrt{\sum_{\alpha, \beta=1}^{p}\left\|\left[A_{\alpha}, A_{\beta}\right]\right\|^{2}}=\frac{1}{n(n-1)} \sqrt{\sum_{\alpha, \beta=1}^{p}\left\|\left[S_{\alpha}, S_{\beta}\right]\right\|^{2}} .
$$

Since, the operators $S_{\alpha}$ are symmetric and trace-free, we can apply the theorem of Lu at any point of $M$ to get

$$
\sum_{\alpha, \beta=1}^{p}\left\|\left[S_{\alpha}, S_{\beta}\right]\right\|^{2} \leqslant\left(\sum_{\alpha=1}^{p}\left\|S_{\alpha}\right\|^{2}\right)^{2}
$$

Thus,

$$
\rho^{\perp} \leqslant \frac{1}{n(n-1)} \sum_{\alpha=1}^{n}\left\|S_{\alpha}\right\|^{2}=\frac{1}{n(n-1)}\|\tau\|^{2} .
$$

Reporting this in 4), we obtain

$$
\|H\|^{2} \geqslant \rho+\rho^{\perp}+\left(\frac{\left(a^{\prime}\right)^{2}}{a^{2}}-\frac{c}{a^{2}}\right)\left(1-\frac{2}{n}\|T\|^{2}\right)-\frac{2 a^{\prime \prime}}{n a}\|T\|^{2},
$$

which concludes the proof.

\section{An APPLiCATiOn to SUbMANifOLdS OF $\mathbb{R} \times_{e^{\lambda t}} \mathbb{H}^{n}(c)$}

We finish this note by the following application of Theorem 1 to submanifolds of warped product of the type $\mathbb{R} \times_{a} \mathbb{H}^{n}(c)$ where $a$ is the real function defined by $a(t)=e^{\lambda t}$ with $\lambda$ a real constant.

Corollary 1. Let $M^{m}$ be a submanifold of the warped product $\mathbb{R} \times_{e^{\lambda t}} \mathbb{H}^{n}(c)$ with normalized scalar and normal scalar curvatures $\rho$ and $\rho^{\perp}$ and mean curvature $H$. Then, we have

$$
\|H\|^{2} \geqslant \rho+\rho^{\perp}+\lambda^{2}-c e^{-2 \lambda t}\left(1-\frac{2}{n}\|T\|^{2}\right)
$$


Proof: This comes directly from Theorem 1 , with the fact that $\frac{\left(a^{\prime}\right)^{2}}{a^{2}}-\frac{c}{a^{2}}=\lambda^{2}-c e^{-2 \lambda t}$ and $\frac{a^{\prime \prime}}{a^{2}}=\lambda^{2}$. Hence the term $\left(\frac{\left(a^{\prime}\right)^{2}}{a^{2}}-\frac{c}{a^{2}}\right)\left(1-\frac{2}{n}\|T\|^{2}\right)-\frac{2 a^{\prime \prime}}{n a}\|T\|^{2}$ becomes $\lambda^{2}-$ $c e^{-2 \lambda t}\left(1-\frac{2}{n}\|T\|^{2}\right)$.

Comparing $\|H\|^{2}$ with $\rho$ is a natural question which leads to rigidity results. Indeed, by the Gauss formula, we know that, for hypersurfaces of space forms, $\rho$ is up to a constant (which is the sectional curvature $k$ of the ambiant space form) the second mean curvature $\mathrm{H}_{2}$, that is the second elementary symmetric polynomial in the prinicpal curvatures. Moreover, it is a classical fact that $H^{2} \geqslant H_{2}$ with equality at umbilical points. Hence, assuming $H^{2} \leqslant \rho-k$ implies that $M$ is a hypersphere. In this spirit, and using the above DDVV inequality, we give the following rigidity result.

Corollary 2. Let $M^{m}$ be a complete submanifold without boundary of the warped product $\mathbb{R} \times_{e^{\lambda t}} \mathbb{H}^{n}(c)$ with normalized scalar and normal scalar curvatures $\rho$ and $\rho^{\perp}$ and mean curvature $H$. If $\|H\|^{2} \leqslant \rho+\lambda^{2}$, then

$$
\|H\|^{2}=\rho+\lambda^{2}, \quad \rho^{\perp}=0, \quad n=2 \quad \text { and } \quad\|T\|=1 .
$$

Hence, $M$ is a surface of the type $\mathbb{R} \times_{e^{\lambda t}} \gamma$, where $\gamma$ is a curve in $\mathbb{H}^{n}(c)$.

Proof: First note that since $n \geqslant 2$ and $\|T\|^{2} \leqslant 1$ and $c<0$, we have $c e^{-2 \lambda t}\left(1-\frac{2}{n}\|T\|^{2}\right) \leqslant$ 0 . Note also that, by defintion, $\rho^{\perp} \geqslant 0$. Hence, from Corollary $1,\|H\|^{2} \leqslant \rho+\lambda^{2}$ is possible if and only if $\|H\|^{2}=\rho+\lambda^{2}, \rho^{\perp}=0, n=2$ and $\|T\|=1$. Since $n=2$, then $M$ is a surface and the fact that $\|T\|=1$ implies that $T=\partial_{t}$ and so $M$ is of the type $I \times e^{\lambda t} \gamma$, where $\gamma$ is a curve in $\mathbb{H}^{n}(c)$. Since we assume that $M$ is complete and without boundary, $I=\mathbb{R}$. This concludes the proof.

\section{REFERENCES}

[1] Q. Chen \& Q. Cui, Normal scalar curvature and a pinching theorem in $\mathbb{S}^{m} \times \mathbb{R}$ and $\mathbb{H}^{m} \times \mathbb{R}$, Science China Math. 54(9) (2011),1977-1984.

[2] De Smet P.J., Dillen F., Verstraelen L. and Vrancken L., A pointwise inequality in submanifold theory, Arch. Math. 35(1) (1999), 115-128.

[3] F. Dillen, J. Fastenakels \& J. Van der Veken, Remarks on an inequality involving the normal scalar curvature, in Pure and Applied Differential Geometry, Aachen: Shaker-Verlag, 2007, 83-92.

[4] J. Q. Ge \& Z. Z. Tang, A proof of the DDVV conjecture and its equality case, Pacific J Math, 237 (2008) 87-95.

[5] M.-A. Lawn \& M. Ortega, A Fundamental Theorem for Hypersurfaces in Semi-Riemannian Warped Products, J. Geom. Phys. 90 (2015), 55-70.

[6] Z. Q. Lu, Normal scalar curvature conjecture and its applications, J Funct Anal, 261 (2011) 1284-1308.

LAMA, UPEM-UPEC-CNRS, CitÉ Descartes, Champs Sur MARne, 77454 Marne-LA-VALlÉE CEDEX 2, FRANCE

E-mail address: julien.roth@u-pem.fr 